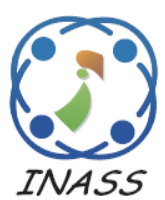

\title{
Adaptive Optimal Trajectory Tracking Control for Four Flexible Joint Robot Manipulator
}

\author{
Randa Jalaa Yahya ${ }^{1 *} \quad$ Nizar Hadi Abbas ${ }^{1}$ \\ ${ }^{1}$ Department of Electrical Engineering, College of Engineering, University of Baghdad, Iraq \\ * Corresponding author's Email: r.zaki1802m@coeng.uobaghdad.edu.iq
}

\begin{abstract}
The Swarm Intelligence Optimization Methods were widely used in science. Animals' swarming behaviour mainly inspires these methods. Conventional optimization for Harris Hawks (HHO) is a recent technique based on the hunting strategy of harris hawks. The parallelism technique was used in this study to try to improve the original version of the algorithm. The improved algorithm sets the parameters of two controllers designed to improve the 4-link flexible joint manipulator's dynamic performance. The integral sliding mode controller controls the flexible joints, and the adaptive output feedback tracking controller controls the rigid links. When the improved algorithm is tested on benchmark functions and compared to other optimization strategies. the results show that the improved algorithm is faster and more precise. The simulation results indicate the improved algorithm's capabilities, with an enhancement percentage of 76.72 percent for the first link and 70.91 percent for the first joint compared to the standard approach.
\end{abstract}

Keywords: Flexible joint manipulator, An integral sliding mode controller, Adaptive output feedback tracking control, Parallel harris hawks optimizer, Harris hawks optimizer.

\section{Introduction}

An important field of research is robotic manipulation. The advancement of manipulator research can be grouped into two types: Rigid Manipulator and Flexible Manipulator (FM). Due to its many benefits over rigid manipulators, current research is oriented to FMs. The FMs benefit from light weight, low cost, reduced size, more workspace, portability and economy [1]. This has led to much research into developing high-performance control approaches using state-of-the-art control theories. For instance, hybrid PID controller [2], model reference adaptive control [3], nonlinear fractionalorder PID [4], adaptive fuzzy sliding mode control [5], Fuzzy logic self-tuning PID control [6] have been dedicated to the study of flexible-joint robots.

An Integral Sliding Mode Controller (ISMC) tracks a flexible joint driven by a DC motor. It is an efficient control strategy for resolving many issues with the Sliding Mode Control (SMC) approach, including the high-frequency chattering effect and insensitivity[7].
All robot manipulator dynamic parameters are assumed to be unknown and the manipulator needs to follow the desired path. Adaptive Output Feedback Tracking Controller (AOFTC) allows robot manipulators to complete tasks in an unknown environment [8], This controller was utilized to control the rigid link in this work.

In this research work, the ISMC \& AOFTC parameters were tuned using the standard Harris Hawks Optimization (HHO) algorithm and a new nature-inspired algorithm named Parallel Harris Hawks Optimization (PHHO) algorithm. Parallel processing aims to produce the same results through multiple processors in order to minimize runtime. This technique was used in different algorithms like Parallel Genetic Algorithm [9], Parallel Particle Swarm Optimization with Communication Strategies [10], and parallel grey wolf optimizer [11].

HHO algorithm [12] is a nature-inspired optimization paradigm inspired by Harris hawks' cooperative behaviour in nature. The HHO has been used widely with mathematical models due to its nature to mimics the dynamic patterns of such models. 
The authors have employed the HHO in various scientific topics such as environmental, medical, and chemical fields. The researchers proposed other techniques to enhance the HHO's performance to benefit its work, i.e. In [13], the authors present the MOHHO algorithm for environmental purposes to estimate air pollutant concentrations by adjusting the extreme learning machine (ELM) model parameters to achieve high precise and stable model. In [14], chaotic maps with HHO (CHHO) algorithm are introduced to improve feature selection of the medical field. Finally, on [15], a cuckoo search with chaotic map $\mathrm{HHO}$ (CHHO-CS) is presented to improve the performance of $\mathrm{HHO}$ that is used with chemical descriptor selection. In this work, compared to [13-15], in this paper, $\mathrm{PHHO}$ presents to increase the manipulator's ability to overcome the trajectory tracking problem by improving both exploration, exploitation, and feature selection speed.

The PHHO performance is tested by benchmark test functions classified as (unimodal, multimodal, and fixed dimension multimodal test functions). The benchmark test functions are used to obtain the average and standard deviation for the improved algorithm to be demonstrated and finally compared to other optimization algorithms: Chaotic Harris Hawks Optimizer (CHHO) [16], HHO, Salp Swarm Algorithm (SSA) [17], Gravitational Search Algorithm (GSA) [18], and Particle Swarm Optimization (PSO) [19].

The robot manipulator with four degrees of freedom is considered. The task is to move the robot manipulator in the workspace along a desire position while minimizing an objective function. The objective function presents the error between the actual and the desired positions. Hence, the aim is to improve an optimization algorithm for tuning the controller parameters that consider the effects of system uncertainties, so the closed-loop system output tracking error converges to zero asymptotically.

The rest of the paper is organized as follows: Section 2 establishes the modelling for four rigid link / flexible joint manipulators. Section 3 presents the theoretical basics for controlling methods and the standard algorithm HHO. Section 4 the improvement of HHO and the flowchart are explored. Section 5 contains the findings and discussion. Finally, section 6 summarizes the findings of this research.

\section{Modeling of four rigid link - flexible joint manipulator}

The general dynamic equation form for a four Rigid Link - Flexible Joint Manipulator (4-RLFJM) is presented in this section, and the state-space form is presented. The dynamics of the model robot manipulator can be described as [20]:

$$
\boldsymbol{\tau}=\mathbf{M}(\boldsymbol{q}) \ddot{\boldsymbol{q}}+\mathbf{C}(\boldsymbol{q}, \dot{\boldsymbol{q}}) \dot{\boldsymbol{q}}+\mathbf{g}(\boldsymbol{q})
$$

Where $\boldsymbol{q}, \dot{\boldsymbol{q}}, \ddot{\boldsymbol{q}} \in \mathbf{R}^{n \times n}$ denote link position, velocity and acceleration vectors, respectively, $\mathbf{M}(\boldsymbol{q})$ $\in \mathbf{R}^{n \times n}$ and $\mathbf{C}(\boldsymbol{q}, \dot{\boldsymbol{q}}) \in \mathbf{R}^{n \times n}$ are manipulator inertia and centrifugal/Coriolis forces, respectively and $\mathbf{g}(\boldsymbol{q}) \in \mathbf{R}^{n}$ is a vector of gravitational forces. Appendix A to show the element of the matrices.

Electric motors have a mechanical subsystem and electrical subsystem, as illustrated in the following dynamic Eqs. (2), and (3), respectively [21]:

$$
\begin{gathered}
\mathrm{J} \ddot{q}_{\mathrm{m}}+\mathrm{B} \dot{q}_{\mathrm{m}}+\mathrm{r} \tau=k_{m} I_{a} \\
L_{m} \dot{I}_{a}+R_{m} I_{a}+K_{b} \dot{q}_{\mathrm{m}}=v(t)
\end{gathered}
$$

where $\boldsymbol{q}_{\mathbf{m}}, \dot{\boldsymbol{q}}_{\mathbf{m}}, \ddot{\boldsymbol{q}}_{\mathbf{m}} \in \mathbf{R}^{n}$ denote Joint position, velocity and acceleration vectors, respectively, $\boldsymbol{J} \in$ $\mathrm{R}^{n \times n}, \mathbf{B} \in \mathbf{R}^{n \times n}$ and $\mathbf{r} \in \mathbf{R}^{n \times n}$ are diagonal matrices to represent coefficients of the motor inertia, motor damping, and reduction gear, respectively. $\boldsymbol{k}_{\boldsymbol{m}} \in \mathrm{R}^{n \times n}$ diagonal matrices for the coefficients of torque of the armature. $\boldsymbol{L}_{\boldsymbol{m}} \in \mathrm{R}^{n \times n}, \quad \boldsymbol{R}_{\boldsymbol{m}} \in$ $\mathrm{R}^{n \times n}$, and $\boldsymbol{K}_{b} \in \mathrm{R}^{n \times n}$ illustrate the $\mathrm{n} \times \mathrm{n}$ diagonal matrices for the coefficients of electrical inductance, armature resistance, and back-EMF constant, respectively. $\boldsymbol{I}_{a} \in \mathbf{R}^{n}$ is a vector of armature current and $\boldsymbol{v}(\boldsymbol{t}) \in \mathbf{R}^{n}$ is the control input voltage applied to the joint actuators. Torsional springs connect the rigid bodies. In this instance, the joint torques $\tau$ are transmitted through the $\mathrm{n}$ shafts:

$$
\tau=K_{s}\left(r q_{m}-q\right)
$$

where $\boldsymbol{k}_{\boldsymbol{s}} \in \mathrm{R}^{n \times n}$ are diagonal matrices to represent coefficients of lumped flexibility.

Then, Eqs. (1) and (2) can be written as follows to obtain the state space representation:

$$
\begin{gathered}
\ddot{\boldsymbol{q}}=\boldsymbol{M}(\boldsymbol{q})^{-1}\left[\boldsymbol{K}_{s}\left(\boldsymbol{r} \boldsymbol{q}_{m}-\boldsymbol{q}\right)-\boldsymbol{C}(\boldsymbol{q}, \dot{\boldsymbol{q}}) \dot{\boldsymbol{q}}-\boldsymbol{g}(\boldsymbol{q})\right] \\
\ddot{\boldsymbol{q}}_{\boldsymbol{m}}=\boldsymbol{J}^{-1}\left(\boldsymbol{\tau}_{m^{-}} \boldsymbol{B} \dot{\boldsymbol{q}}_{\boldsymbol{m}}-\boldsymbol{r} \boldsymbol{K}_{s}\left(\boldsymbol{r} \boldsymbol{q}_{\boldsymbol{m}}-\boldsymbol{q}\right)\right)
\end{gathered}
$$

\section{Theoretical basics}

\subsection{Integral sliding mode control}

ISMC's main concept is high-frequency switching gain, which forces the state to achieve the integral sliding surface [7]. The states are then guided 
to the desired equilibrium point by the integral action in the sliding manifold. It reduces chattering and improving the control system's robustness and accuracy while maintaining nominal control efficiency. The sliding manifold selected for the system is [22]:

$$
s=\dot{e}+c e+k_{i} \int \dot{e}+c e \mathrm{~d} t
$$

where $\mathbf{c}$ and $\mathbf{k}_{\mathbf{i}}$ are positive design parameters and $\mathbf{e}$ is the error value between the desired and actual angular positions $\left(\mathbf{q}_{\mathbf{d}}\right)$ and $(\mathbf{q})$, respectively,

$$
\boldsymbol{e}=\boldsymbol{q}_{d}-\boldsymbol{q} ; \dot{\boldsymbol{e}}=\dot{\boldsymbol{q}}_{\boldsymbol{d}}-\dot{\boldsymbol{q}}, \text { and } \boldsymbol{e}=\ddot{\boldsymbol{q}}_{\mathbf{d}}-\ddot{\boldsymbol{q}}
$$

where $\boldsymbol{q}_{\boldsymbol{d}}$ represents the joint positions that are desired. The control signal consists of two terms that are the equivalent $\left(\mathbf{u}_{\text {eq }}\right)$ and the switching $\left(\mathbf{u}_{\text {sw }}\right)$ control laws, depicts through:

$$
\boldsymbol{u}=\boldsymbol{u}_{e q}+\boldsymbol{u}_{s w}
$$

where the equivalent law is given by,

$$
u_{e q i}=w_{i} \int \dot{e}+c e d t
$$

where $\boldsymbol{w}_{\boldsymbol{i}}$ is a positive design parameter.

Also, the design of the switching law is given by,

$$
\boldsymbol{u}_{s w i}=\boldsymbol{k}_{\boldsymbol{i}} \operatorname{sgn}\left(\boldsymbol{s}_{\boldsymbol{i}}\right)
$$

Then, the control law $\boldsymbol{u}$ is important for driving the system's initial states to equilibrium in a finite time.

$$
u_{I S M C}=k_{i} \operatorname{sgn}\left(s_{i}\right)+w_{i} \int \dot{e}+c e d t
$$

\subsection{Adaptive output feedback tracking control}

The adaptive controller for a four-link manipulator system with unknown manipulator parameters is formulated. A control law for actuator torques and an estimation law for unknown parameters have been developed. The manipulator output $\boldsymbol{q}$ tracks the desired trajectories $\boldsymbol{q}_{\boldsymbol{d}}$ after an initial adaptation process [8].

\subsubsection{Control law}

The control law can be defined to design an adaptive controller:

$$
\boldsymbol{u}_{\text {AOFTC }}=\underset{K_{p} \tanh (\sigma e)}{Y\left(q_{d}, \dot{q}_{d}, \ddot{q}_{d}\right) \widehat{\theta}+K_{v} \tanh (\widetilde{\varphi})+}
$$

where

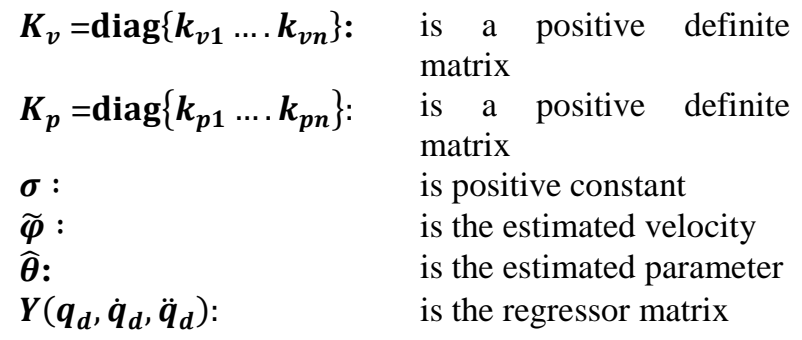

The estimated velocity $\widetilde{\boldsymbol{\varphi}}$ in the adaptive controller (1) is obtained from the following nonlinear filter:

$$
\begin{aligned}
& \dot{\boldsymbol{x}}=\boldsymbol{A} \tanh (\widetilde{\boldsymbol{\varphi}}) \\
& \widetilde{\boldsymbol{\varphi}}=\boldsymbol{x}+\boldsymbol{B} \boldsymbol{e}
\end{aligned}
$$

where $\quad \mathrm{A}=\quad \operatorname{diag}\left\{a_{1} \ldots . a_{v n}\right\} \quad$ and $\mathrm{B}=\operatorname{diag}\left\{b_{1} \ldots . b_{p n}\right\}$ are positive definite matrices.

\subsubsection{Analysis}

The adaptation parameters of the robot are calculated as follows for adaptive controller analysis (Appendix B to Show the theoretical derivation).:

$$
\begin{aligned}
& \widehat{\boldsymbol{\theta}} \\
& =\Gamma\left[\boldsymbol{Y}^{T}\left(\boldsymbol{q}_{d}, \dot{\boldsymbol{q}}_{d}, \ddot{\boldsymbol{q}}_{\boldsymbol{d}}\right) \boldsymbol{e}\right. \\
& -\int_{\mathbf{0}}^{t}\left\{\dot{\boldsymbol{Y}}^{T}\left(\boldsymbol{q}_{d}, \dot{\boldsymbol{q}}_{d}, \ddot{\boldsymbol{q}}_{\boldsymbol{d}}\right) \boldsymbol{e}-\xi \boldsymbol{Y}^{T}\left(\boldsymbol{q}_{d}, \dot{\boldsymbol{q}}_{\boldsymbol{d}}, \ddot{\boldsymbol{q}}_{\boldsymbol{d}}\right) \tanh (\boldsymbol{\sigma e})\right\} \boldsymbol{d t}
\end{aligned}
$$

where $\boldsymbol{\Gamma}$ is a positive definite matrix and $\xi \in$ $\left(\xi_{\min }, \xi_{\max }\right)$ the strictly positive constant.

For forming a closed-loop system, the control law was given in Eq. (13) is replaced in the dynamic equation of robots given in Eq. (1). The estimation error parameter is specified:

$$
\widetilde{\boldsymbol{\theta}}=\boldsymbol{q}-\widehat{\boldsymbol{\theta}} \in \mathbb{R}^{r}
$$

\subsection{Standard harris hawks optimizer}

The Harris Hawks Optimizer is a new metaheuristic algorithm to solve problems of global optimization. Generally, the HHO mimics the activities of hawks in nature while hunting for and catching prey. The HHO searches for two stages (exploration and exploitation), employing a variety of methods. The following are the symbols used in this algorithm to make HHO easier to understand [23] : 1. Vector of Hawks position (search agents) $\boldsymbol{X}_{\boldsymbol{i}}(\boldsymbol{t})$.

2. Position of Rabbit (best agent) $\boldsymbol{X}_{\text {rabbit }}(\boldsymbol{t})$.

3. Position of a random Hawk $\boldsymbol{X}_{\text {rand }}(\boldsymbol{t})$.

4. Hawks average position $\boldsymbol{X}_{\boldsymbol{m}}(\boldsymbol{t})$. 
5. Maximum number of iterations, swarm size, iteration counter $\mathrm{T}, \mathrm{N}, \mathrm{t}$.

6. Random numbers between $(0,1) \boldsymbol{r}_{1}, \boldsymbol{r}_{2}, \boldsymbol{r}_{3}, \boldsymbol{r}_{4}, \boldsymbol{r}_{5}, \boldsymbol{q}$, $u, v$.

7. Dimension, lower and upper bounds of variables $\boldsymbol{D}$, $L B, U B$.

8. Initial state of energy, escaping energy $\boldsymbol{E}_{\boldsymbol{0}}, \boldsymbol{E}$.

9. The difference between the position vector of the rabbit and the current location $\Delta \boldsymbol{X}(\boldsymbol{t})$.

10. The rabbit's random jump strength during the escape method $\mathrm{J}$.

\subsubsection{Exploration phase}

It is defined as:

$$
\begin{aligned}
& X(t+1)= \\
& \left\{\begin{array}{c}
X_{\text {rand }}(t)-r_{1}\left|X_{\text {rand }}(t)-2 r_{2} X(t)\right| \\
q \geq 0.5 \\
\left(X_{\text {rabbit }}(t)-X_{m}(t)\right)- \\
r_{3}\left(L B+r_{4}(U B-L B)\right) \quad q<0.5
\end{array}\right.
\end{aligned}
$$

The average location of the Hawks $X_{m}$ is represented by

$$
X_{m}(t)=\frac{1}{N} \sum_{i=1}^{N} X_{i}(t)
$$

\subsubsection{Transition from exploration to exploitation}

The HHO algorithm can change from exploration to exploitation and switch between several exploitative behaviors, depending on the prey's escape energy. During the escape behavior, a prey's energy level plummets dramatically. A rabbit's energy is modelled as follows:

$$
E=2 E_{0}\left(1-\frac{t}{T}\right)
$$

\subsubsection{Exploitation phase}

\section{Case1: Soft besiege}

When $r \geq 0.5$ and $|E| \geq 0.5$, the rabbit still has enough energy to attempt to escape by making a series of random, deceptive hops. The Harris' hawks encircle the rabbit softly during their attempts to tire it and then perform the surprise pounce.

$$
\begin{gathered}
X(t+1)=\Delta X(t)-E\left|J X_{\text {rabbit }}(t)-X(t)\right| \\
\Delta X(t)=X_{\text {rabbit }}(t)-X(t)
\end{gathered}
$$

\section{Case2: Hard besiege}

When $r \geq 0.5$ and $|E|<0.5$, the prey is completely exhausted, with very little escaping energy. In addition, the Harris' hawks hardly surround the intended prey to perform the surprise hit finally

$$
X(t+1)=X_{\text {rabbit }}(t)-E|\Delta X(t)|
$$

\section{Case3: Soft besiege with progressive rapid dives}

When still $|E| \geq 0.5$ but $r<0.5$, even though the rabbit has enough stamina to escape successfully, a soft besiege is built before the surprise pounce. The Lévy Flights (LF) are started here to emulate Hawk and rabbit's various movements.

$$
\begin{gathered}
Y=X_{\text {rabbit }}(t)-E\left|J X_{\text {rabbit }}(t)-X(t)\right| \\
Z=Y+S \times L F(D)
\end{gathered}
$$

where $S$ is the size $1 \mathrm{xD}$ random vector and LF is the levy flight function,

$$
\begin{aligned}
& L F(x)=0.01 \times \frac{u \times \sigma}{|v|^{\frac{1}{\beta}}}, \\
& \sigma=\left(\frac{\Gamma(1+\beta) \times \sin \left(\frac{\pi \beta}{2}\right)}{\left.\Gamma\left(\frac{1+\beta}{2}\right) \times \beta \times 2\left(\frac{\beta-1}{2}\right)\right)}\right)^{\frac{1}{\beta}}
\end{aligned}
$$

In the end, the position of the hawks should be updated using:

$$
X(t+1)= \begin{cases}Y & \text { if } F(Y)<F(X(t)) \\ Z & \text { if } F(Z)<F(X(t))\end{cases}
$$

where $\mathrm{Y}$ and $\mathrm{Z}$ are obtained using equations.

Case4: Hard besiege with progressive rapid dives

When $|E|<0.5$ and $r<0.5$, because the rabbit's ability to escape is insufficient, a hard siege is recommended before carrying out a series of surprise attacks to capture and kill the prey.in this step, hawks attempt to lower the varied distances between their prey and the average position.

$$
X(t+1)= \begin{cases}Y & \text { if } F(Y)<F(X(t)) \\ Z & \text { if } F(Z)<F(X(t))\end{cases}
$$

By applying new rules, new $\boldsymbol{Y}$ and $\boldsymbol{Z}$ values are proposed. Eqs. (28) and (29) where $\boldsymbol{X}_{\boldsymbol{m}}(\boldsymbol{t})$ is obtained using Eq. (18)

$$
\begin{gathered}
Y=X_{\text {rabbit }}(t)-E\left|J X_{\text {rabbit }}(t)-X_{m}(t)\right| \\
Z=Y+S \times L F(D)
\end{gathered}
$$

Fig.1 illustrates all phases of HHO [12]. 


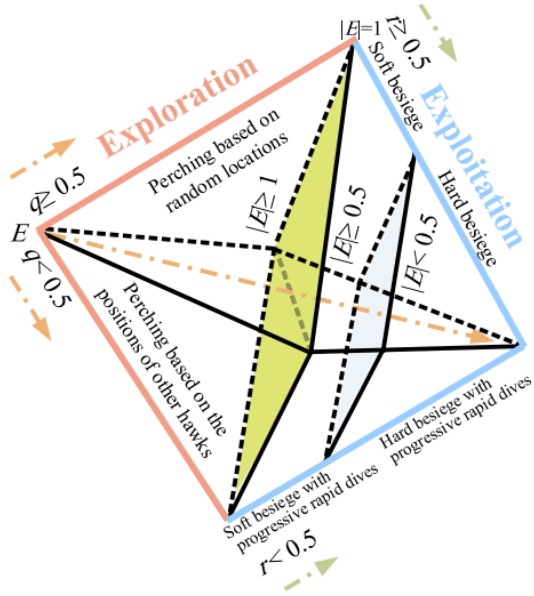

Figure .1 Shows all phases of HHO

\section{Proposed parallel optimizer}

The purpose of parallel processing is to provide the same results using several processors to reduce the processing time. Pipeline processing and data parallelism are the two most common parallel processing methods [11]. This technique has been used in different algorithms such as Parallel Genetic Algorithm or Parallel Particle Swarm Optimization with communication Strategies. At first, the Harris hawks have two or more sub-groups divided into. Each subgroup is implemented independently based on the main structure of the algorithm as well as the results are shared each time the iteration happens. Fig. 2 shows the procedure for the improved algorithm.

\section{Numerical results and discussions}

\subsection{Test functions details}

Twelve benchmark problems were used to measure PHHO's ability in this part. The benchmark functions are a set of five unimodal functions have used (F01-F04), while five multimodal functions were used (F05-F08), and finally, fixed dimension multimodal functions were used (F09-F12).

\subsection{Proposed algorithm evaluation}

In comparison to the twelve test functions chosen, the proposed algorithm was evaluated with other optimization algorithms, like CHHO [16], HHO [12], SSA [17], GSA [18], PSO [19]. The mean and standard deviation were determined after each test was run 30 times for a maximum of 500 iterations. In Fig. 3, the performance of PHHO against other optimization algorithms is shown. Table 1 shows the results of the chosen benchmarking functions.

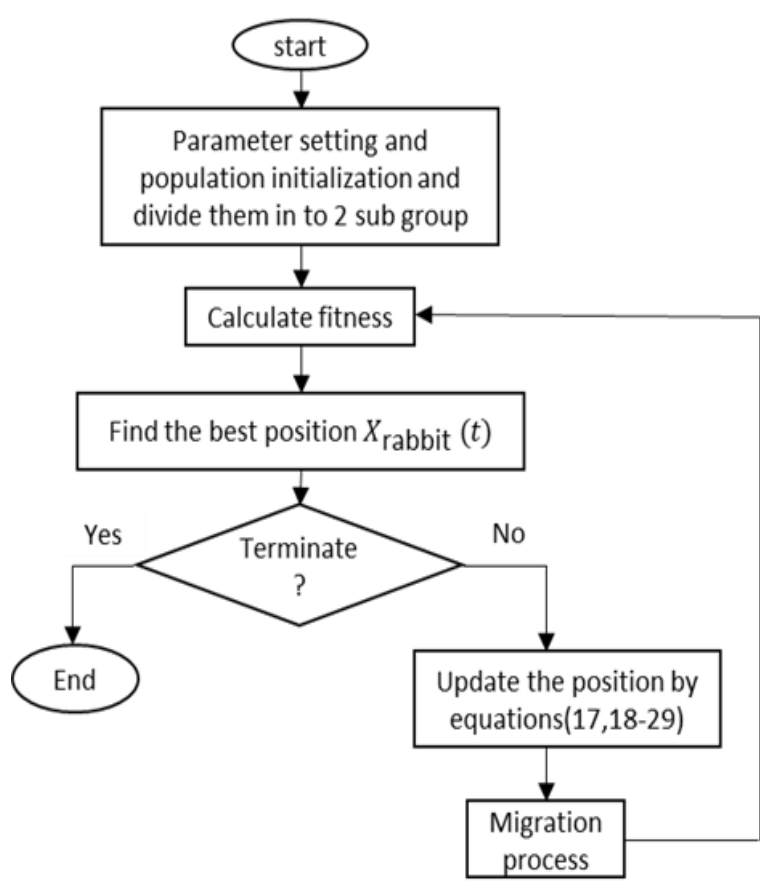

Figure. 2 Flowchart of the improved algorithm

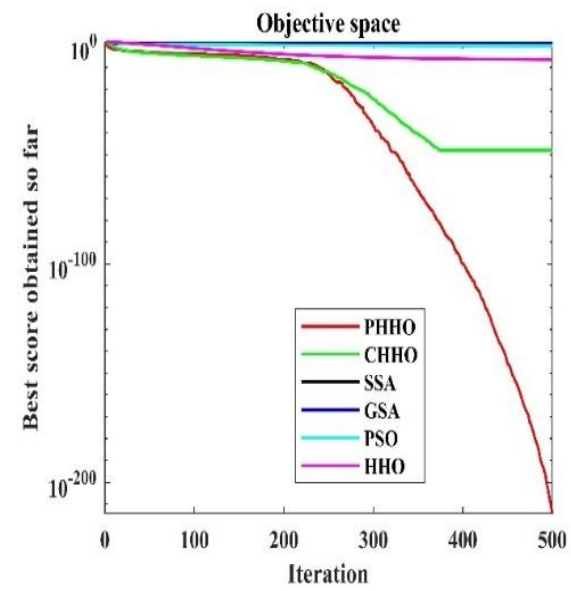

(a)

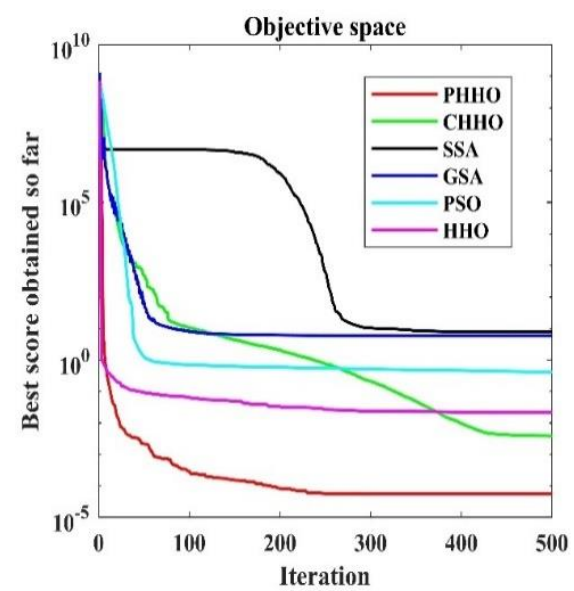

(b)

Figure. 3 Performance comparison of algorithms based on the selected test functions: (a) F3(Schwefel 2.21) and (b) F8(Generalized Penalized) 
Table 1. Comparison of PHHO with $\mathrm{CHHO}, \mathrm{HHO}$, SSA, GSA, PSO.

\begin{tabular}{|c|c|c|c|}
\hline Function & algorithms & avg & std \\
\hline \multirow{6}{*}{$\begin{array}{c}\text { F1 } \\
\text { Sphere }\end{array}$} & PHHO & 0 & 0 \\
\hline & CHHO & $2.779 * 10^{-95}$ & $1.379 * 10^{-9}$ \\
\hline & HHO & $1.056 * 10^{-27}$ & $1.296 * 10^{-2}$ \\
\hline & SSA & $1.189 * 10^{-6}$ & $1.086 * 10^{-6}$ \\
\hline & GSA & $1.579 * 10^{-3}$ & $6.737 * 10^{-4}$ \\
\hline & PSO & $1.969 * 10^{-3}$ & $2.113 * 10^{-4}$ \\
\hline \multirow{6}{*}{$\begin{array}{c}\text { F2 } \\
\text { step }\end{array}$} & PHHO & 0 & 0 \\
\hline & СНHO & $5.649 * 10^{-52}$ & $3.024 * 10^{-5}$ \\
\hline & HHO & $3.045^{*} 10^{-5}$ & $1.218 * 10^{-4}$ \\
\hline & SSA & $1.026 * 10^{4}$ & $4.962 * 10^{3}$ \\
\hline & GSA & $1.007 * 10^{3}$ & $3.693 * 10^{2}$ \\
\hline & PSO & $7.804 * 10$ & $6.062 * 10$ \\
\hline \multirow{6}{*}{$\begin{array}{c}\text { F3 } \\
\text { Schwefel } \\
2.21\end{array}$} & PHНO & $7.49 * 10^{-215}$ & 0 \\
\hline & СНHO & $1.397 * 10^{-48}$ & $6.234 * 10^{-48}$ \\
\hline & HHO & $8.309 * 10^{-7}$ & $9.969 * 10^{-7}$ \\
\hline & SSA & $2.901 * 10$ & 7.160330 \\
\hline & GSA & 7.253 & 1.807 \\
\hline & PSO & 1.1439 & $2.758 * 10^{-1}$ \\
\hline \multirow{6}{*}{$\begin{array}{c}\text { F4 } \\
\text { Schwefel } \\
2.22\end{array}$} & PHHO & $8.48 * 10^{-214}$ & 0 \\
\hline & CHHO & $7.481 * 10^{-40}$ & $6.954 * 10^{-4 c}$ \\
\hline & HHO & $3.071 * 10^{-41}$ & $1.403 * 10^{-4 c}$ \\
\hline & SSA & $5.707 * 10$ & $3.505 * 10$ \\
\hline & GSA & $1.451 * 10^{-1}$ & $4.417 * 10^{-1}$ \\
\hline & PSO & $3.054 * 10^{-2}$ & $2.564 * 10^{-2}$ \\
\hline \multirow{6}{*}{$\begin{array}{c}\text { F5 } \\
\text { Rastrigin }\end{array}$} & PHНO & $1.376 * 10^{-3}$ & $2.294 * 10^{-3}$ \\
\hline & CHHO & $2.725 * 10^{-1}$ & $2.725 * 10^{-1}$ \\
\hline & HНO & $2.793 * 10$ & 4.256 \\
\hline & SSA & $3.169 * 10^{2}$ & $6.868 * 10^{2}$ \\
\hline & GSA & $8.719 * 10$ & $5.428 * 10^{2}$ \\
\hline & PSO & $7.249 * 10$ & $8.433 * 10$ \\
\hline \multirow{6}{*}{$\begin{array}{c}\text { F6 } \\
\text { Ackley }\end{array}$} & PHHO & $8.882 * 10^{-16}$ & 0 \\
\hline & СНHО & $1.025 * 10^{-13}$ & $1.881 * 10^{-1}$ \\
\hline & НHO & $8.882 * 10^{-16}$ & 0 \\
\hline & SSA & 6.445 & 1.673 \\
\hline & GSA & $3.104 * 10^{-2}$ & $1.701 * 10^{-1}$ \\
\hline & PSO & $2.735^{*} 10^{-1}$ & $5.297 * 10^{-1}$ \\
\hline \multirow{6}{*}{$\begin{array}{c}\text { F7 } \\
\text { Penalized 1 }\end{array}$} & PHHO & $7.836 * 10^{-05}$ & $9.664 * 10^{-5}$ \\
\hline & CHHO & $6.419 * 10^{-3}$ & $9.871 * 10^{-3}$ \\
\hline & HHO & $2.227 * 10^{-3}$ & $2.025 * 10^{-3}$ \\
\hline & SSA & $2.087 * 10$ & 7.802 \\
\hline & GSA & 1.776 & 1.234 \\
\hline & PSO & $3.459 * 10^{-3}$ & $2.893 * 10^{-2}$ \\
\hline \multirow{6}{*}{$\begin{array}{c}\text { F8 } \\
\text { Generalize } \\
\text { d } \\
\text { Penalized }\end{array}$} & PHHO & $9.493 * 10^{-5}$ & $1.281 * 10^{-4}$ \\
\hline & СНHO & $3.618 * 10^{-2}$ & $3.256 * 10^{-2}$ \\
\hline & HHO & $6.413 * 10^{-1}$ & $1.245 * 10^{-1}$ \\
\hline & SSA & $1.407 * 10$ & $1.169 * 10$ \\
\hline & GSA & 1.023 & 7.045 \\
\hline & PSO & $6.424 * 10^{-3}$ & $6.163 * 10^{-3}$ \\
\hline \multirow{6}{*}{$\begin{array}{c}\text { F9 } \\
\text { Shekel's } \\
\text { Foxholes }\end{array}$} & PHHO & 1.328 & $9.467 * 10^{-1}$ \\
\hline & СНHО & 4.228 & 4.257 \\
\hline & HНO & 3.719 & 2.991 \\
\hline & SSA & 2.824 & 2.165 \\
\hline & GSA & 6.295 & 4.554 \\
\hline & PSO & 2.874 & 2.165 \\
\hline F10 & PHHO & -3.322 & $1.521 * 10^{-15}$ \\
\hline
\end{tabular}

International Journal of Intelligent Engineering and Systems, Vol.15, No.2, 2022

\begin{tabular}{|c|c|c|c|}
\hline Hartman 5 & CHHO & -3.183 & $6.347 * 10^{-2}$ \\
\cline { 2 - 4 } & HHO & -2.265 & $1.426^{*} 10^{-1}$ \\
\cline { 2 - 4 } & SSA & -3.223 & $5.633^{*} 10^{-2}$ \\
\cline { 2 - 4 } & GSA & -3.101 & $1.443^{*} 10^{-1}$ \\
\cline { 2 - 4 } & PSO & -3.291 & $5.348^{*} 10^{-2}$ \\
\hline \multirow{4}{*}{ F11 } & PHHO & -9.143 & 2.344 \\
\cline { 2 - 4 } Shekel 5 & CHHO & -7.761 & 2.441 \\
\cline { 2 - 4 } & HHO & -5.033 & $2.079 * 10^{-2}$ \\
\cline { 2 - 4 } & SSA & -6.064 & 3.503 \\
\cline { 2 - 4 } & GSA & -6.665 & 3.792 \\
\cline { 2 - 4 } & PSO & -6.713 & 3.192 \\
\hline \multirow{4}{*}{$\begin{array}{c}\text { F12 } \\
\text { Shekel 10 }\end{array}$} & PHHO & -9.169 & 2.081 \\
\cline { 2 - 4 } & CHHO & $-6.009 * 10$ & 1.689 \\
\cline { 2 - 4 } & HHO & -5.237 & $5.299 * 10^{-1}$ \\
\cline { 2 - 4 } & SSA & -4.779 & 3.862 \\
\cline { 2 - 4 } & GSA & $-1.031 * 10$ & 1.284 \\
\cline { 2 - 4 } & PSO & -5.931 & 2.747 \\
\hline \multicolumn{2}{|c|}{} & \\
\hline
\end{tabular}

According to the above, there is only one local minima value for unimodal test functions, but multimodal test functions have multiple local minima values as the number of task dimensions increases [24]. However, based on the above result, it is clear that unimodal test functions (F1, F2, F3, and F4), multimodal test functions (F5, F6, F7, and F8), and fixed multimodal test functions (F9, F10, F11, and F12) tend to reach towards more optimal minimum value than that $\mathrm{HHO}$ and SSA. At the same time, it nearly converges (F10), but it has a lower minimum amount than HHO and SSA.

\subsection{Model results and discussions}

Results from simulations in MATLAB/ Simulink are used to check the efficiency and efficiency of a system of the suggested algorithm for tracking control of four-link flexible joint manipulators. The system's dynamic equation and parameter values are provided in Table 2 to evaluate the system's performance with a population size of 30 , max iteration $=200$, lower bound $=-300$, and upper bound $=300$.

The gains in controls are optimized by optimizing the system's dynamic response. First, the standard HHO was used to tune the controllers' parameters, then compared with the PHHO tuning results. Fig. 4 shows the robot response by AOFTC - HHO with AOFTC - PHHO for a) $1^{\text {st }}$ link, b) $2^{\text {nd }}$ link,

Table 2. Parameters of the system [21, 25]

\begin{tabular}{|c|c|c|c|}
\hline Parameters & Value & Parameters & Value \\
\hline $\boldsymbol{B}$ & 0.1 & $\boldsymbol{K}_{\boldsymbol{s}}$ & 4 \\
\hline $\boldsymbol{K}_{\boldsymbol{b}}$ & 0.01 & $\boldsymbol{J}$ & 0.01 \\
\hline $\boldsymbol{K}_{\boldsymbol{m}}$ & 0.01 & $\mathbf{L}$ & 0.34 \\
\hline $\boldsymbol{R}_{\boldsymbol{m}}$ & 1 & $\mathbf{m}$ & 1.51 \\
\hline $\mathbf{L}_{\mathbf{m}}$ & 0.5 & $\mathbf{g}$ & 9.81 \\
\hline
\end{tabular}


c) $3^{\text {rd }}$ link, and d) $4^{\text {th }}$ link positions. Fig. 5 illustrates the robot manipulator response by ISMC - HHO and

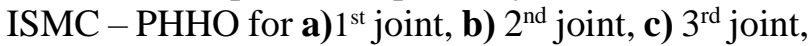
and d) $4^{\text {th }}$ joint positions.

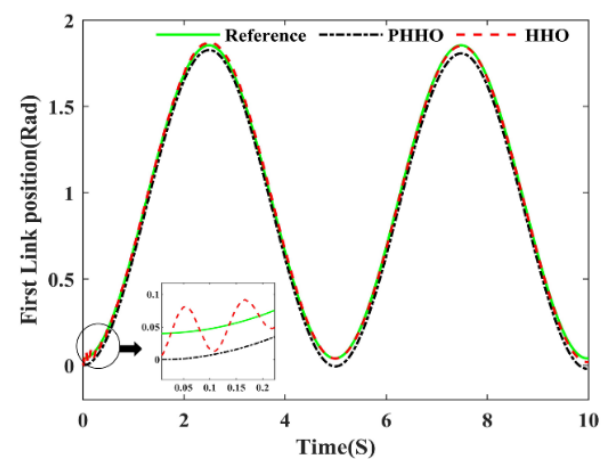

(a)

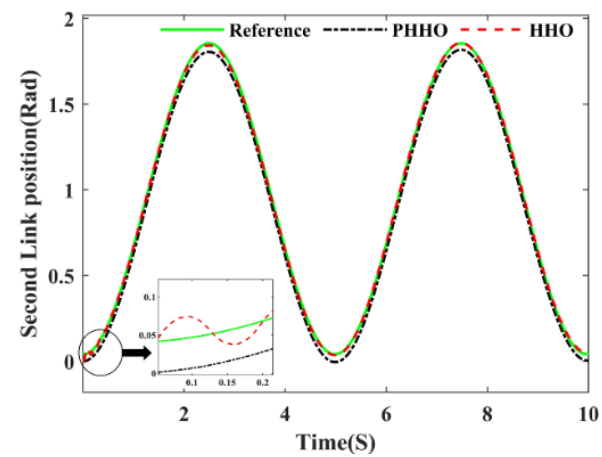

(b)

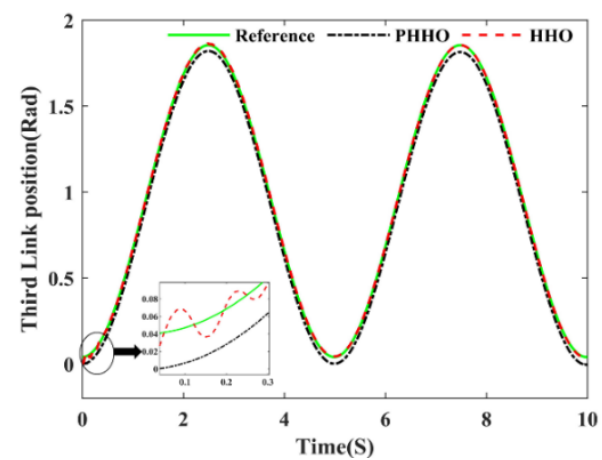

(c)

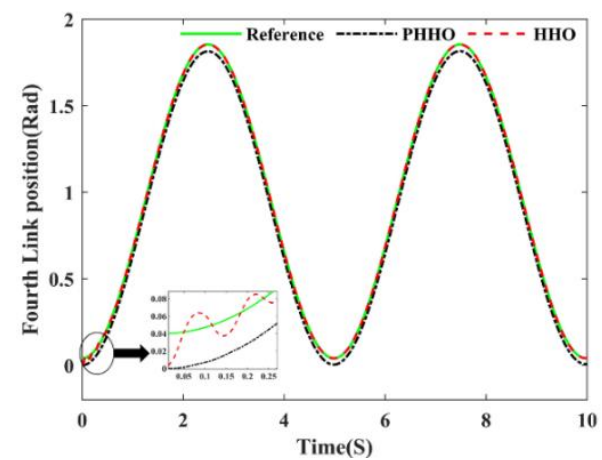

(d)

Figure. 4 Position tracking results of: (a) $1^{\text {st }} \operatorname{link}$, (b) $2^{\text {nd }}$ link, (c) $3^{\text {rd }}$ link, and (d) $4^{\text {th }}$ link controlled by AOFTCPHHO \& AOFTC-HHO

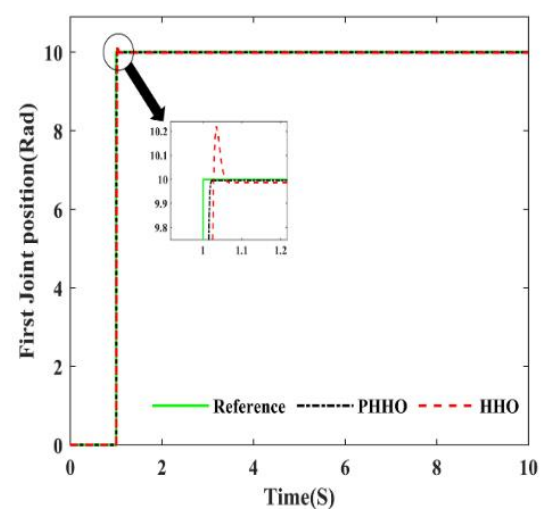

(a)

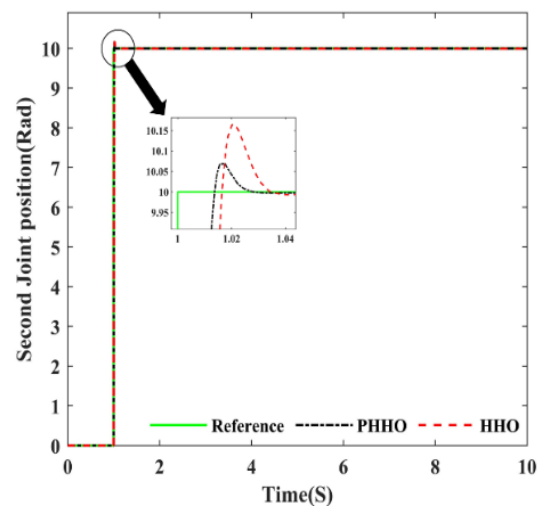

(b)

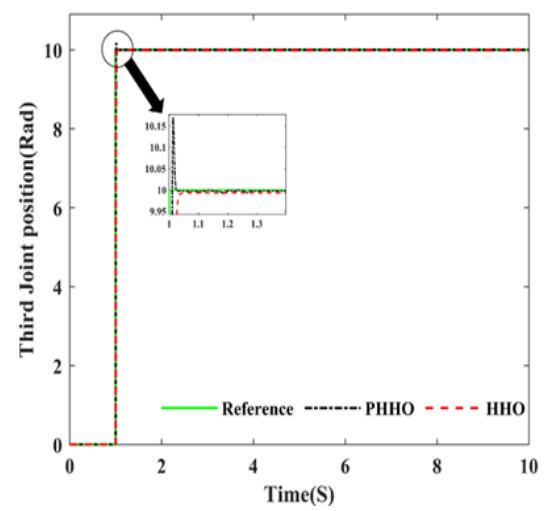

(c)

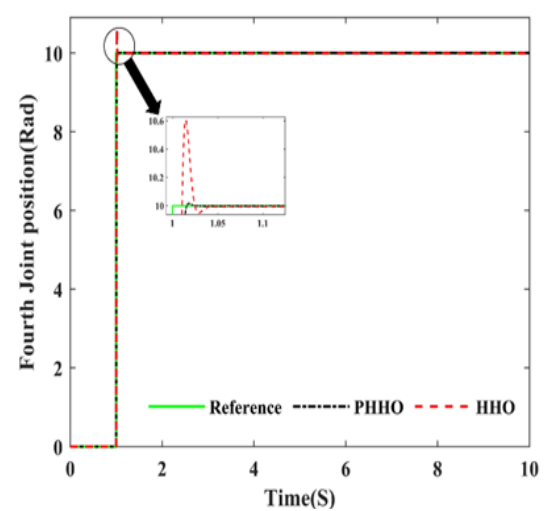

(d)

Figure. 5 Position tracking results of: (a) $1^{\text {st }}$ joint, (b) $2^{\text {nd }}$ joint, (c) $3^{\text {rd }}$ joint, and (d) $4^{\text {th }}$ joint controlled by ISMC-

PHHO \& ISMC-HHO 
Table 3. Cost function (controllers-PHHO)

\begin{tabular}{|l|l|l|l|}
\hline Theta1 & 0.0149 & Thetam1 & 0.1862 \\
\hline Theta2 & 0.0664 & Thetam2 & 0.1201 \\
\hline Theta3 & 0.0346 & Thetam3 & 0.1201 \\
\hline Theta4 & 0.0104 & Thetam4 & 0.0903 \\
\hline
\end{tabular}

Table 4. Cost function (controllers-HHO)

\begin{tabular}{|l|l|l|l|}
\hline Theta1 & 0.064 & Thetam1 & 0.64 \\
\hline Theta2 & 0.105 & Thetam2 & 0.378 \\
\hline Theta3 & 0.098 & Thetam3 & 0.342 \\
\hline Theta4 & 0.0497 & Thetam4 & 0.325 \\
\hline
\end{tabular}

The better position is where the objective function is lower, also known as the fitness function. The ITAE performance criterion is the cost function that must be minimized. The criterion of the integral of the absolute magnitude of error (ITAE) has the following definition [25]:

$$
I T A E=\int_{0}^{T} t|e(t)| d t
$$

The results obtained in Table 4 and Table 5 clearly show that the controllers tuned by PHHO present a better cost function than the typical HHO, which present lower performance for $1^{\text {st }}$ link position (Theta1) and $1^{\text {st }}$ joint position (Thetam1), $2^{\text {nd }}$ link position (Theta2) and $2^{\text {nd }}$ joint position (Thetam2), $3^{\text {rd }}$ link position (Theta3) and $3^{\text {rd }}$ joint position (Thetam3), and the same indication observed for $4^{\text {th }}$ link position (Theta4) and $4^{\text {th }}$ joint position (Thetam4).

The enhancement percentage can be calculated according to the following equation:

$$
\begin{gathered}
E P]_{\text {algorithm A over algorithm } B} \\
100 \%
\end{gathered}
$$

$E P$ described the enhancement percentage, while $O B$ described the objective function value of each algorithm. Then, the enhancement of PHHO, which is the new version of $\mathrm{HHO}$ over its original algorithm, is:

$$
\begin{gathered}
E P]_{A O F C-P H H O} \text { over } A O F C-H H O \\
100 \%=76.72 \%\left(\text { for } 1^{\text {st }}\right. \text { link position) } \\
E P]_{I S M C-P H H O \text { over } I S M C-H H O}=\left(1-\frac{0.0149}{0.686}\right) \times \\
100 \%=70.91 \% \text { (for } 1^{\text {st }} \text { joint position) }
\end{gathered}
$$

The same procedure can be used on the remaining joints and links, with the following results: $36.76 \%, 64.69 \%, 78.78 \%$ for second, third, fourth link positions, respectively, and $68.23 \%, 96.49 \%$, $72.22 \%$ for the second, third, fourth joint positions.

\section{Conclusions}

In this paper, an improved algorithm called PHHO tuners is proposed to determine the optimal design parameters of controllers used for tracking control of robot manipulators. Compared to HHO and other algorithms, experimental results showed the developed algorithm is more efficient in delivering high-quality solutions with rational computational iteration (unimodal, multimodal, and fixed dimensions multimodal. The enhancement percentage indicates that the responses obtained from the controllers that tuned by PHHO optimization are better compared to the results obtained from the controllers that tuned by $\mathrm{HHO}$ optimization by $76.72 \%$ and $70.91 \%$ for first link and joint respectively, as well as for the rest of the links and the joints as illustrated above. In the future, the authors plan to the experimental implementation of this work on a real robot.

\section{Conflicts of Interest}

The authors declare no conflict of interest.

\section{Author Contributions}

Randa Jalaa contributed to methodology, software, and writing review and editing, Nizar Hadi contributed in supervising the overall work and editing the paper.

\section{Reference}

[1] K. Lochan and B. K. Roy, "Position control of two-link flexible manipulator using low chattering SMC techniques", Int. J. Control Theory Appl., Vol. 8, No. 3, pp. 1137-1145, 2015.

[2] K. Ibrahim and A. B. Sharkawy, "A hybrid PID control scheme for flexible joint manipulators and a comparison with sliding mode control", Ain Shams Engineering Journal, Vol. 9, No. 4, pp. 3451-3457, 2018.

[3] T. Pourseif, M. T. Andani, Z. Ramezani, and M. Pourgholi, "Model Reference Adaptive Control for Robot Tracking Problem: Design \& Performance Analysis", International Journal of Control Science and Engineering, Vol. 7, No. 1, pp. 18-23, 2017.

[4] A. Kumar, P. J. Gaidhane, and V. Kumar, "A nonlinear fractional order PID controller applied to redundant robot manipulator", In: Proc. of 
2017 6th Int. Conf. Comput. Appl. Electr. Eng. Recent Adv. CERA 2017, pp. 527-532, 2018.

[5] D. Gao, Z. Sun, and W. Wang, "Adaptive fuzzy sliding mode control for robotic manipulators", In: Proc. of 2010 8th World Congress on Intelligent Control and Automation, pp. 48114816, 2010.

[6] A. Dehghani and H. Khodadadi, "Fuzzy Logic Self-Tuning PID control for a single-link flexible joint robot manipulator in the presence of uncertainty", In: Proc. of ICCAS 2015 - 2015 15th Int. Conf. Control. Autom. Syst. Proc., pp. 186-191, 2015.

[7] W. Alam, A. Mehmood, K. Ali, U. Javaid, S. Alharbi, and J. Iqbal, "Nonlinear control of a flexible joint robotic manipulator with experimental validation", Journal of Mechanical Engineering, Vol. 64, No. 1, pp. 4755, 2018.

[8] J. Moreno and S. Gonzalez, "An Adaptive Output Feedback Tracking Controller for Manipulators Subject to Constrained Torques", In: Proc. of the 45th IEEE Conference on Decision and Control, pp. 2026-2031, 2006.

[9] H. Gehring, and A. Bortfeldt, "A Parallel Genetic Algorithm for Solving the Container Loading Problem", International Transactions in Operational Research, Vol. 9, No. 4, pp. 497$511,2002$.

[10] J. F. Chang, S. C. Chu, J. F. Roddick, and J. S. Pan, "A parallel particle swarm optimization algorithm with communication strategies", Journal of Information Science and Engineering, Vol. 21, No. 4, pp. 809-818, 2005.

[11] M. S. Nasrabadi, Y. Sharafi, and M. Tayari, "A parallel grey wolf optimizer combined with opposition based learning", In: Proc. of 2016 1st Conference on Swarm Intelligence and Evolutionary Computation, pp. 18-23, 2016.

[12] A. A. Heidari, S. Mirjalili, H. Faris, I. Aljarah, M. Mafarja, and H. Chen, "Harris hawks optimization: Algorithm and applications", Future Generation Computer Systems, Vol. 97, pp. 849-872, 2019.

[13] P. Du, J. Wang, Y. Hao, T. Niu, and W. Yang, "A novel hybrid model based on multi-objective Harris hawks optimization algorithm for daily PM2.5 and PM10 forecasting", Applied Soft Computing, Vol. 96, p. 106620, 2020.

[14] Z. M. Elgamal, N. B. M. Yasin, M. Tubishat, M. Alswaitti, and S. Mirjalili, "An improved harris hawks optimization algorithm with simulated annealing for feature selection in the medical field", IEEE Access, Vol. 8, No. 8, pp. 186638-
186652, 2020.

[15] E. H. Houssein, M. E. Hosney, M. Elhoseny, D. Oliva, W. M. Mohamed, and M. Hassaballah, "Hybrid Harris hawks optimization with cuckoo search for drug design and discovery in chemoinformatics", Scientific Reports, Vol. 10, No. 1, pp. 1-22, 2020.

[16] T. Singh, "A chaotic sequence-guided Harris hawks optimizer for data clustering", Neural Computing and Applications, Vol. 32, No. 23, pp. 17789-17803, 2020.

[17] S. Mirjalili, A. H. Gandomi, S. Z. Mirjalili, S. Saremi, H. Faris, and S. M. Mirjalili, "Salp Swarm Algorithm: A bio-inspired optimizer for engineering design problems", Advances in Engineering Software, Vol. 114, pp. 163-191, 2017.

[18] E. Rashedi, H. N. pour, and S. Saryazdi, "GSA: A Gravitational Search Algorithm", Information Sciences, Vol. 179, No. 13, pp. 2232-2248, 2009.

[19] R. Poli, J. Kennedy, and T. Blackwell, "Particle swarm optimization: An overview", Swarm Intelligence, Vol. 1, No. 1, pp. 33-57, 2007.

[20] M. L. Kevin and C. P. Frank, Modern Robotics: Mechanics, Planning, and Control. Cambridge: Cambridge University Press, 2017.

[21] M. R. Soltanpour, S. Zaare, M. Haghgoo, and M. Moattari, "Free-Chattering Fuzzy Sliding Mode Control of Robot Manipulators with Joints Flexibility in the presence of Matched and Mismatched Uncertainties in Model Dynamic and Actuators", Journal of Intelligent \& Robotic Systems, Vol. 100, No. 1, pp. 47-69, 2020.

[22] Y. Shtessel, C. Edwards, L. Fridman, and A. Levant, Sliding Mode Control and Observation. Control Engineering, 2014.

[23] S. Yasear and K. K. Mahamud, "Fine-Tuning the Ant Colony System Algorithm Through Harris's Hawk Optimizer for Travelling Salesman Problem", International Journal of Intelligent Engineering and Systems, Vol. 14, No. 4, pp. 136-145, 2021.

[24] M. W. Hasan and N. H. Abbas, "Controller design for underwater robotic vehicle based on improved whale optimization algorithm", Bull. Electr. Eng. Informatics, Vol. 10, No. 2, pp. 609618, 2021.

[25] D. Maiti, A. Acharya, M. Chakraborty, A. Konar, and R. Janarthanan, "Tuning PID and $\mathrm{PI}^{\lambda} \mathrm{D}^{\delta}$; Controllers using the Integral Time Absolute Error Criterion", In: Proc. of 2008 4th Int. Conf. Inf. Autom. Sustain., pp. 1-6, 2008. 


\section{Appendix A}

The model for the four-link manipulator was derived from the two-link manipulator concept [20] as follows:

$$
\begin{aligned}
& {\left[\begin{array}{l}
\tau_{1} \\
\tau_{2} \\
\tau_{3} \\
\tau_{4}
\end{array}\right]=\left[\begin{array}{llll}
M_{11} & M_{12} & M_{13} & M_{14} \\
M_{21} & M_{22} & M_{23} & M_{24} \\
M_{31} & M_{32} & M_{33} & M_{34} \\
M_{41} & M_{42} & M_{43} & M_{44}
\end{array}\right]\left[\begin{array}{l}
\ddot{q}_{1} \\
\ddot{q}_{2} \\
\ddot{q}_{3} \\
\ddot{q}_{4}
\end{array}\right]+} \\
& {\left[\begin{array}{l}
C_{1} \\
C_{2} \\
C_{3} \\
C_{4}
\end{array}\right]\left[\begin{array}{l}
\dot{q}_{1} \\
\dot{q}_{2} \\
\dot{q}_{3} \\
\dot{q}_{4}
\end{array}\right]+\left[\begin{array}{l}
G_{1} \\
G_{2} \\
G_{3} \\
G_{4}
\end{array}\right]}
\end{aligned}
$$

The elements of the matrices $\mathbf{M}, \mathbf{C}$, and $\mathbf{G}$ in equation A.1 are defined as:

$$
\begin{aligned}
& M_{11}=\mu_{1} L_{1}^{2}+\mu_{2} L_{2}^{2}+\mu_{3} L_{3}^{2}+\mu_{4} L_{4}^{2}+2 \mu_{2} v_{1} \\
& +2 \mu_{3}\left(v_{2}+v_{3}\right)+2 \mu_{4}\left(v_{4}+v_{5}+v_{6}\right) \\
& M_{12}=M_{21}=\mu_{2} L_{2}^{2}+\mu_{3} L_{3}^{2}+\mu_{4} L_{4}^{2}+\mu_{2} v_{1} \\
& +\mu_{3}\left(v_{2}+2 v_{3}\right)+\mu_{4}\left(v_{4}+2 v_{5}+2 v_{6}\right) \\
& M_{13}=M_{31}=\mu_{3} L_{3}^{2}+\mu_{4} L_{4}^{2}+\mu_{3}\left(v_{2}+v_{3}\right)+\mu_{4}\left(v_{4}\right. \\
& \left.+v_{5}+2 v_{6}\right) \\
& M_{14}=M_{41}=\mu_{4} L_{4}^{2}+\mu_{4}\left(v_{4}+v_{5}+v_{6}\right) \\
& M_{22}=\mu_{2} L_{2}^{2}+\mu_{3} L_{3}^{2}+\mu_{4} L_{4}^{2}+2 \mu_{3} v_{3}+2 \mu_{4}\left(v_{5}\right. \\
& \left.+v_{6}\right) \\
& M_{23}=M_{32}=\mu_{3} L_{3}^{2}+\mu_{4} L_{4}^{2}+\mu_{3} v_{3}+\mu_{4}\left(v_{5}+2 v_{6}\right) \\
& M_{24}=M_{42}=\mu_{4} L_{4}^{2}+\mu_{4}\left(v_{5}+v_{6}\right) \\
& M_{33}=\mu_{3} L_{3}^{2}+\mu_{4} L_{4}^{2}+2 \mu_{3} v_{3}+2 \mu_{4} v_{6} \\
& M_{34}=M_{43}=\mu_{4} L_{4}^{2}+\mu_{4} v_{6} \\
& M_{44}=\mu_{4} L_{4}^{2} \\
& G_{1}=\mu_{1} L_{1} g c_{1}+\mu_{2} L_{2} g c_{12}+\mu_{3} L_{3} g c_{123} \\
& +\mu_{4} L_{4} g c_{1234} \\
& G_{2}=\mu_{2} L_{2} g c_{12}+\mu_{3} L_{3} g c_{123}+\mu_{4} L_{4} g c_{1234} \\
& G_{3}=\mu_{3} L_{3} g c_{123}+\mu_{4} L_{4} g c_{1234} \\
& G_{4}=\mu_{4} L_{4} g c_{1234} \\
& \mathrm{C}_{1}=-\mu_{2} \mathrm{~L}_{1} \mathrm{~L}_{2} S_{2}\left(2 \dot{q}_{1} \dot{q}_{2}+\dot{q}_{2}^{2}\right) \\
& -\mu_{3} \mathrm{~L}_{1} \mathrm{~L}_{3} S_{23}\left(\dot{q}_{2}+\dot{q}_{3}\right) \\
& \left(2 \dot{q}_{1}+\dot{q}_{2}+\dot{q}_{3}\right)-\mu_{3} \mathrm{~L}_{2} \mathrm{~L}_{3} S_{3}\left(\dot{q}_{3}\right)\left(2 \dot{q}_{1}+2 \dot{q}_{2}+\dot{q}_{3}\right) \\
& -\mu_{4} \mathrm{~L}_{1} \mathrm{~L}_{4} S_{234}\left(\dot{q}_{2}+\dot{q}_{3}+\dot{q}_{4}\right)\left(2 \dot{q}_{1}+\dot{q}_{2}+\dot{q}_{3}\right. \\
& \left.+\dot{q}_{4}\right) \\
& -\mu_{4} \mathrm{~L}_{2} \mathrm{~L}_{4} S_{34}\left(\dot{q}_{3}+\dot{q}_{4}\right)\left(2 \dot{q}_{1}+2 \dot{q}_{2}+\dot{q}_{3}+\dot{q}_{4}\right) \\
& -\mu_{4} \mathrm{~L}_{3} \mathrm{~L}_{4} S_{4}\left(\dot{q}_{4}\right)\left(2 \dot{q}_{1}+2 \dot{q}_{2}+2 \dot{q}_{3}+\dot{q}_{4}\right) \\
& \mathrm{C}_{2}=\mu_{2} \mathrm{~L}_{1} \mathrm{~L}_{2} S_{2}\left(\dot{q}_{1}^{2}\right)+\mu_{3}\left[\mathrm{~L}_{1} \mathrm{~L}_{3} S_{23}\left(\dot{q}_{1}^{2}\right)-\mathrm{L}_{2} \mathrm{~L}_{3}\right. \\
& \left.S_{3}\left(\dot{q}_{3}\right)\left(2 \dot{q}_{1}+2 \dot{q}_{2}+\dot{q}_{3}\right)\right]+\mu_{4}\left[\mathrm{~L}_{1} \mathrm{~L}_{4} S_{234}\left(\dot{q}_{1}^{2}\right)\right. \\
& -\mathrm{L}_{2} \mathrm{~L}_{4} S_{34}\left(\dot{q}_{3}+\dot{q}_{4}\right)\left(2 \dot{q}_{1}+2 \dot{q}_{2}+\dot{q}_{3}+\dot{q}_{4}\right) \\
& \left.-\mathrm{L}_{3} \mathrm{~L}_{4} S_{4}\left(\dot{q}_{4}\right)\left(2 \dot{q}_{1}+2 \dot{q}_{2}+2 \dot{q}_{3}+\dot{q}_{4}\right)\right] \\
& \mathrm{C}_{3}=\mu_{3}\left[\mathrm{~L}_{1} \mathrm{~L}_{3} S_{23}\left(\dot{q}_{1}^{2}\right)+\mathrm{L}_{2} \mathrm{~L}_{3} S_{3}\left(\dot{q}_{1}+\dot{q}_{2}\right)^{2}\right] \\
& +\mu_{4}\left[\mathrm{~L}_{1} \mathrm{~L}_{4} S_{234}\left(\dot{q}_{1}^{2}\right)+\mathrm{L}_{2} \mathrm{~L}_{4} S_{34}\left(\dot{q}_{1}+\dot{q}_{2}\right)^{2}\right. \\
& \left.-\mathrm{L}_{3} \mathrm{~L}_{4} S_{4}\left(\dot{q}_{4}\right)\left(2 \dot{q}_{1}+2 \dot{q}_{2}+2 \dot{q}_{3}+\dot{q}_{4}\right)\right] \\
& \mathrm{C}_{4}=\mu_{4} \mathrm{~L}_{1} \mathrm{~L}_{4} S_{234}\left(\dot{q}_{1}^{2}\right)+\mu_{4} \mathrm{~L}_{2} \mathrm{~L}_{4} S_{34}\left(\dot{q}_{1}+\dot{q}_{2}\right)^{2} \\
& +\mu_{4} \mathrm{~L}_{3} \mathrm{~L}_{4} S_{4}\left(\dot{q}_{1}+\dot{q}_{2}+\dot{q}_{3}\right)^{2}
\end{aligned}
$$

International Journal of Intelligent Engineering and Systems, Vol.15, No.2, 2022 where,

$$
\begin{aligned}
& \mu_{1}=\mathrm{m}_{1}+\mathrm{m}_{2}+\mathrm{m}_{3}+\mathrm{m}_{4} \\
& \mu_{2}=\mathrm{m}_{2}+\mathrm{m}_{3}+\mathrm{m}_{4} \\
& \mu_{3}=\mathrm{m}_{3}+\mathrm{m}_{4} \\
& \mu_{4}=\mathrm{m}_{4} \\
& c_{1}=\cos \left(q_{1}\right), c_{12}=\cos \left(q_{1}+q_{2}\right) \ldots \\
& S_{1}=\sin \left(q_{1}\right), S_{12}=\sin \left(q_{1}+q_{2}\right) \ldots \\
& v_{1}=\mathrm{L}_{1} \mathrm{~L}_{2} \cos \left(q_{2}\right) \\
& v_{2}=\mathrm{L}_{1} \mathrm{~L}_{3} \cos \left(q_{2}+q_{3}\right) \\
& v_{3}=\mathrm{L}_{2} \mathrm{~L}_{3} \cos \left(q_{3}\right) \\
& v_{4}=\mathrm{L}_{1} \mathrm{~L}_{4} \cos \left(q_{2}+q_{3}+q_{4}\right) \\
& v_{5}=\mathrm{L}_{2} \mathrm{~L}_{4} \cos \left(q_{3}+q_{4}\right) \\
& v_{6}=\mathrm{L}_{3} \mathrm{~L}_{4} \cos \left(q_{4}\right)
\end{aligned}
$$

\section{Appendix B}

Also, the controller for the four-link manipulator was derived based on the two link manipulator [8], then let the parameter be:

$$
\begin{aligned}
& \mathrm{R}_{1}=\left(\mathrm{m}_{1}+\mathrm{m}_{2}+\mathrm{m}_{3}+\mathrm{m}_{4}\right) \mathrm{L}_{1}^{2} \\
& \mathrm{R}_{2}=\left(\mathrm{m}_{2}+\mathrm{m}_{3}+\mathrm{m}_{4}\right) \mathrm{L}_{2}^{2} \\
& \mathrm{R}_{3}=\left(\mathrm{m}_{3}+\mathrm{m}_{4}\right) \mathrm{L}_{3}^{2} \\
& \mathrm{R}_{4}=\left(\mathrm{m}_{4}\right) \mathrm{L}_{4}^{2} \\
& \mathrm{R}_{5}=\left(\mathrm{m}_{2}+\mathrm{m}_{3}+\mathrm{m}_{4}\right) \mathrm{L}_{1} \mathrm{~L}_{2} \\
& \mathrm{R}_{6}=\left(\mathrm{m}_{3}+\mathrm{m}_{4}\right) \mathrm{L}_{1} \mathrm{~L}_{3} \\
& \mathrm{R}_{7}=\left(\mathrm{m}_{3}+\mathrm{m}_{4}\right) \mathrm{L}_{2} \mathrm{~L}_{3} \\
& \mathrm{R}_{8}=\left(\mathrm{m}_{4}\right) \mathrm{L}_{1} \mathrm{~L}_{4} \\
& \mathrm{R}_{9}=\left(\mathrm{m}_{4}\right) \mathrm{L}_{2} \mathrm{~L}_{4} \\
& \mathrm{R}_{10}=\left(\mathrm{m}_{4}\right) \mathrm{L}_{3} \mathrm{~L}_{4} \\
& \mathrm{R}_{11}=\left(\mathrm{m}_{1}+\mathrm{m}_{2}+\mathrm{m}_{3}+\mathrm{m}_{4}\right) \mathrm{L}_{1} \\
& \mathrm{R}_{12}=\left(\mathrm{m}_{2}+\mathrm{m}_{3}+\mathrm{m}_{4}\right) \mathrm{L}_{2} \\
& \mathrm{R}_{13}=\left(\mathrm{m}_{3}+\mathrm{m}_{4}\right) \mathrm{L}_{3} \\
& \mathrm{R}_{14}=\left(\mathrm{m}_{4}\right) \mathrm{L}_{4}
\end{aligned}
$$

Sub. Equations (B.1) into the element of the matrices $(D, C, G)$ to get the regressor matrix that consisting of four rows and fourteen columns in:

$$
\begin{aligned}
& \mathbf{Y}\left(\mathbf{q}_{\mathbf{d}}, \dot{\mathbf{q}}_{\mathbf{d}}, \ddot{\mathbf{q}}_{\mathbf{d}}\right) \\
& \quad=\left[\begin{array}{ccccc}
a_{11} & a_{12} & \ldots & a_{113} & a_{114} \\
\vdots & & \ddots & \vdots \\
a_{41} & a_{42} & \cdots & a_{413} & a_{414}
\end{array}\right]_{4 \times 14} \\
& a_{11}=\ddot{q}_{1} \\
& a_{12}=a_{22}=\ddot{q}_{1}+\ddot{q}_{2} \\
& a_{13}=a_{23}=a_{33}=\ddot{q}_{1}+\ddot{q}_{2}+\ddot{q}_{3} \\
& a_{14}=a_{24}=a_{34}=a_{44}=\ddot{q}_{1}+\ddot{q}_{2}+\ddot{q}_{3}+\ddot{q}_{4} \\
& a_{15}=\left(2 \ddot{q}_{1}+\ddot{q}_{2}\right) C_{2}-\left(2 \dot{q}_{1} \dot{q}_{2}+\dot{q}_{2}^{2}\right) S_{2} \\
& a_{16}=\left(2 \ddot{q}_{1}+\ddot{q}_{2}+\ddot{q}_{3}\right) C_{23}- \\
& \left(\dot{q}_{2}+\dot{q}_{3}\right)\left(2 \dot{q}_{1}+\dot{q}_{2}+\dot{q}_{3}\right) S_{23} \\
& a_{17}=a_{27}=\left(2 \ddot{q}_{1}+2 \ddot{q}_{2}+\ddot{q}_{3}\right) C_{3}-\left(\dot{q}_{3}\right)
\end{aligned}
$$




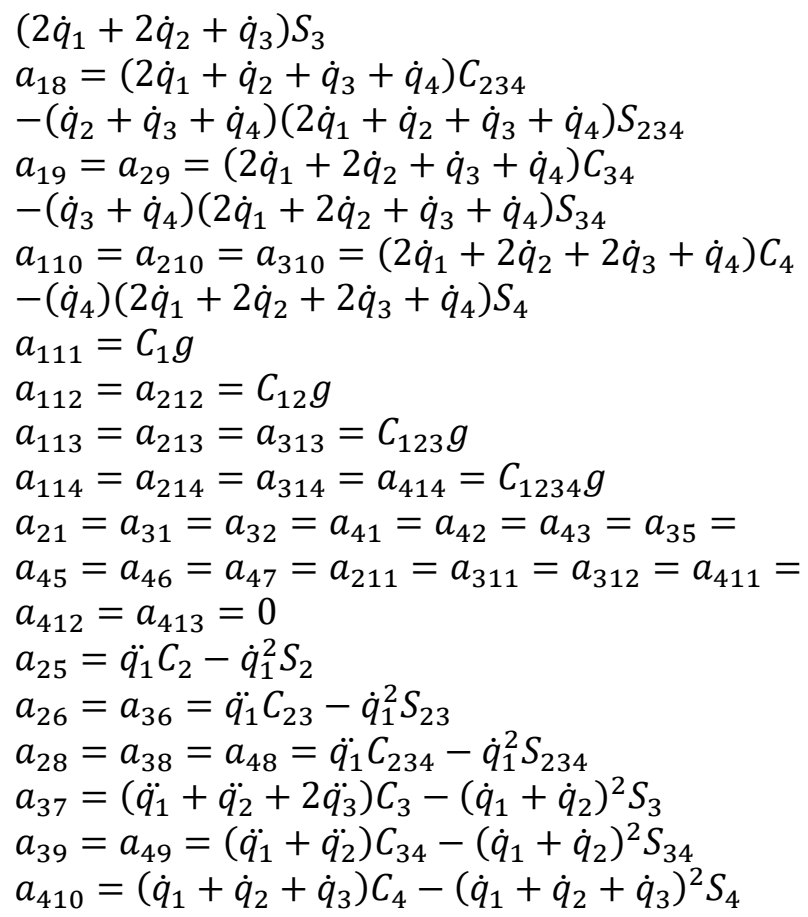

